\title{
AN AXIOMATIC CATEGORISATION FRAMEWORK FOR THE DYNAMIC ALIGNMENT OF DISPARATE FUNCTIONS IN CYBER-PHYSICAL SYSTEMS
}

\author{
Byrne, Thomas J (1,2); Doikin, Aleksandr (1,2); Campean, Felician (1,2); Neagu, Daniel $(1,2)$ \\ 1: University of Bradford, Faculty of Engineering and Informatics; 2: Advanced Automotive Analytics \\ Research Centre
}

\begin{abstract}
Advancing Industry 4.0 concepts by mapping the product of the automotive industry on the spectrum of Cyber Physical Systems, we immediately recognise the convoluted processes involved in the design of new generation vehicles. New technologies developed around the communication core (IoT) enable novel interactions with data. Our framework employs previously untapped data from vehicles in the field for intelligent vehicle health management and knowledge integration into design. Firstly, the concept of an inter-disciplinary artefact is introduced to support the dynamic alignment of disparate functions, so that cyber variables change when physical variables change. Secondly, the axiomatic categorisation (AC) framework simulates functional transformations from artefact to artefact, to monitor and control automotive systems rather than components. Herein, an artefact is defined as a triad of the physical and engineered component, the information processing entity, and communication devices at their interface. Variable changes are modelled using AC, in conjunction with the artefacts, to aggregate functional transformations within the conceptual boundary of a physical system of systems.
\end{abstract}

Keywords: Cyber-Physical System, Big data, Information management, Integrated product development, Knowledge management

Contact:

Byrne, Thomas J

University of Bradford

Engineering and Informatics

United Kingdom

t.j.byrne@bradford.ac.uk

Cite this article: Byrne, T.J., Doikin, A., Campean, F., Neagu, D. (2019) 'An Axiomatic Categorisation Framework for the Dynamic Alignment of Disparate Functions in Cyber-physical Systems', in Proceedings of the 22nd International Conference on Engineering Design (ICED19), Delft, The Netherlands, 5-8 August 2019. DOI:10.1017/dsi.2019.365 


\section{INTRODUCTION}

New generation automotive vehicles have the capability to disseminate previously untapped empirical determinations locally, e.g. for Integrated Vehicle Healthcare Management (IVHM), and/or remotely, for Knowledge Integration into Design. By mapping the product of the automotive industry on the spectrum of cyber-physical systems (CPSs), disparate disciplinary factions are found as the source of opposing trends in design activities and concepts. Two principal sources of authority coexist throughout the lifecycle of a cyber-physical product: one focussed on the design and analysis of physical and engineering systems; the other, focused on information processing and communication technologies (ICT). In this paper, the broad spectrum of CPS challenges are discussed through the viewpoint of the product lifecycle, while mindful that the "product" for disparate design factions is not the teleological automotive vehicle released to the customer, rather the design of parts that make that complex whole. This paper aims to define the constituents for particular use in new generation automotive product design that specify, analyse, verify, validate, monitor and control the physical and engineered system for (i) IVHM (SAE, 2018); (ii) Knowledge Integration into Design - and product development, manufacturing and retirement processes.

A main contribution is the concept of the inter-disciplinary artefact, composed of multidisciplinary constituents by addressing the multidisciplinary design problem with the conceptual inter-disciplinary trinity of the physical and engineering component; the information processing entity; and the communication apparatus at their interface. The nucleus of the proposed inter-disciplinary artefact is the communication core, built from the level of smart devices, data over the air (DOTA), and Internet of Things (IoT) technology. At the core of the research underpinning the results presented in this paper stands the ontological decomposition of objects: conceptual and concrete; temporal and static state; part-of/whole mereology; and the real world matter of substance that has mass and occupies space. The specific scope for this paper is the conceptualisation of concrete matters of substance, part-of/whole relationships in physical and engineered systems and its structure and state for IVHM - notwithstanding the foregoing conclusion that inter-disciplinary artefacts are the exclusive instrument of concrete systems, and that other forms of conceptual objects exist (e.g. for knowledge integration into design application, whereby relationships are exclusively conceptual rather than concrete and physical).

The inter-disciplinary artefact represents the object of perception in CPSs, that is, the trinity of constituent parts: (a) the object in itself, (b) the perceived object, and (c) the instruments used to animate and interact with the objects. At the conceptual design level, the physical component and the information processing entity commune through signals from smart devices (sensors, controllers, and actuators) at their interface. Together, the three constituent parts make up the inter-disciplinary artefact, merging from disparate disciplines at the conceptual design level towards a radical joint individual artefact.

This paper builds on the Automotive Analytics Factory (AAF) framework (Figure 1) previously introduced in Byrne et al (2018). The Automotive Analytics Factory integrates engineering model(s) with data and data-based model(s), where data is the digital footprint of the automotive CPS as it delivers its function(s) within data modelling and knowledge-based processes. The AAF builds on the Product Development Factory, adding data processing and data mining with specific aims to define flexibly data processing cycles that will service specific automotive analytics processes: Diagnostics, Prognostics, Personalised Vehicle Health Management, Knowledge Design Integration and Decision Support.

Within this paper, the AAF is discussed in relation to its application in an industrial context, to:

1. Enhance the operational phase of the product lifecycle using lifetime optimisation models as close to real time IVHM of current generation automotive vehicles in the field.

2. Enhance interconnectedness of stakeholders and the intellectual capital for business, design, manufacturing and retirement processes to help industry make better, faster and more informed decisions through knowledge integration into design and development of new generation products.

The paper discusses the gaps in the research literature in relation to the CPS decomposition (Section 2), followed by the introduction of the Axiomatic Categorisation (AC) framework as a fundamental approach to delivering inter-disciplinary functional requirements (Section 3). A case study of an engine exhaust aftertreatment is considered to illustrate and substantiate with example the implementation of the AC framework (Section 4). In Section 5, we discuss the implications of the 
substantive integration of the CPS paradigm to the design of the substructure architecture of the AAF, introducing a radical new paradigm for the product lifecycle landscape.

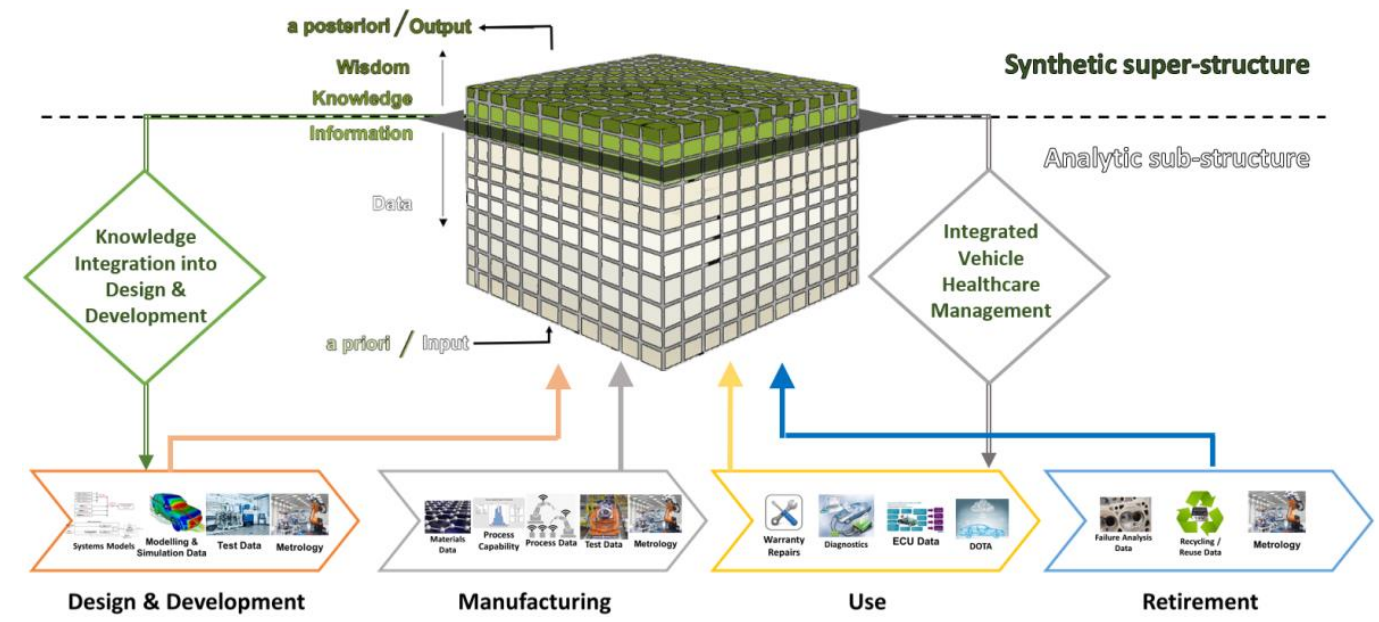

Figure 1. The Automotive Analytics Factory framework

\section{CYBER-PHYSICAL SYSTEM DECOMPOSITION}

The multidisciplinary field of CPS is especially susceptible to new technology forging gaps in design and research. In literature, design activities generally partition at the physical level and the ICT level. To a lesser extent the communication core is singled out (Rajkumar et al., 2010). For a detailed decomposition of important and significant ontological differences between systems see (Dori and Silllitto, 2017). This paper addresses the univocal gap at the conceptual design level whereby the physical system is largely well defined, while information processing systems are in principle defined by how well they describe real world, while in practice descriptions are exclusively domain specific.

CPS decomposition is regarded as a consolidation of multidisciplinary systems (Yan et al., 2017; Raikumar et al., 2010; Wynn et al., 2007), a sophisticated integration of disparate engineering disciplines, and the dependency of iterations upon complex interactions between unpredictable and domain-specific influences. Stankovic (2014) identified five prominent research technologies in the automotive industry: IoT, mobile computing, pervasive computing, wireless senor networks, and CPSs. Hehenberger et al., (2016) defined constituents at the level of IoT, as currently ill- or undefined. IoT technology is a radical new component of the communication core - the kernel to the interdisciplinary artefact, that is, in particular application towards open network requirements.

Multidisciplinary collaboration is needed for the continuous and iterative design and development of both new generation products, and front-end software solutions for current generation products. Developed offline as an AAF activity (see Figure 4 - 4), transferred online over the air towards vehicles in the field, and intended for offline localised computer consumption, the lifecycle of IVHM models will evolve and redevelop in unprecedented iterations, compared to the (10 -15 year) lifecycle of an automotive product as a whole (albeit less for certain sub-systems \& components). By our estimation, IVHM models will evolve at regular intervals, unlike the cyber level representation (digital twin) of physical components that make up the complex whole, that is, the set of inter-disciplinary artefacts, front-end ad-hoc IVHM models will service on top of a digital twin, which is defined and modelled in terms of the function and flow in physical systems.

\subsection{Overview of function and flow in physical systems}

As a discipline, mechanical engineering commonly denotes a branch of engineering focused on the application of Newtonian physics to the analysis and design of machines. In literature, the basis for the "classic" design theory and methodology of Hubka and Eder (1988), guides towards the conceptualisation, definition, and understanding a physical product or system in terms of function. Later developments adopted the Pahl and Beitz's (1988) flows of material, energy, and signal, which underpin the basic principles of Stone and Wood (2000) function modelling, which is widely used across mechanical and electrical engineering, as well as mechatronic systems development, and product service systems design. Otto and Wood (2001) introduced "module heuristics" to support the 
practical implementation of the "functional basis" approach. Functional decomposition of overall functions, in terms of webs of auxiliary functions, was the foundation for Hirtz et al. (2002) functional basis for engineering design which compares earlier function representations with the functional basis, through the integration of those aforementioned independent efforts.

The main function of an automotive powertrain system describes the main components that generate power and deliver it to the road surface. There is a need to consolidate the definition of function across the conceptual landscape, this framework is developed from the constituent level up, at the communication core, where function is the mutual response of the physical and engineered system to propel the vehicle, and that of ICT to specify, analyse, verify, validate, monitor and control physical and engineered systems. In literature, function, behaviour, structure, and state define physical and engineered systems in assorted order and preference (e.g. Yildirim et al., 2017; Ally \& Ning, 2015; Eisenbart et al., 2015; Umeda \& Tomiyana, 2015). We take the position that behaviour is simply the teleological result of function - what remains then is the structure and state of physical systems design, that is, the static state void of dynamic propositions.

\subsection{Cyber level representations of physical systems}

The confusion of natural language is particularly problematic in multidisciplinary design. It permeates from the conceptual design level through to design, manufacturing, implementation, and operational processes. For example, formerly, information processing objects passed messages (see Wand et al., 2000). More recently, object-oriented (O-O) constructs have adopted real world attributes. Recker and Niehaves (2008) describe the theoretical foundation of conceptual models, in its essence, as an investigation of how well a modelling object faithfully and accurately describes objects in the real world. The world however, is susceptible to a near infinite number of interpretations, hence information processing systems are required to do more than what we tell them to do, because what we perceive can be categorised in an almost infinite number of ways. Therefore, CPS design in any social context must have the wherewithal to construct a reality from an entirely a priori set of predicates.

At the conceptual modelling level, designers are frequently confused about whether to show an association between things through a relationship, an entity, or an attribute. Associations in software design (e.g. O-O) and away from conceptual modelling, use association classes, not the associations themselves, as mutual properties to enable message passing. Across disciplines: Umeda et al. (2012) describes entities as components; Dori (2009) denotes objects; Goel et al. (1996 - 2008) refers to substances and components; while Wand and Weber (1990 - 2004) refer to "things", sometimes entities, and associations among things which have a vague reference to "relationships". Pre-millennial efforts (e.g. Goel et al., 1996; Wand and Weber, 1990; Bylander, 1988) towards domain independent constructs were replaced with domain specific models. The problem with domain specificity is not the ambiguity that may exist within the complex whole of a CPS, that is, the conceptual union or artefact of the physical, information, and communication constituents; nor is it the disconnect between the complex whole of a set of artefacts working as part of an integrated system; rather it is the conceptual fallacy between sets of independent complex wholes, or independent systems of systems that interact outside the conceptual boundary of the complex whole or individual CPS. Domain specificity in any social context is only ever an exercise in the pre-conceptual design of that social construct. Add to the multidisciplinary design process, the temporal manifold, and conceptual fallacies of object association, functional non-functional relationships, component or substance, function or behaviour grow exponentially. This research aims to introduce first an Analytic sub-structure construct based on certain original concepts and certain judgements derived from them that must have their origin entirely a priori, independent from empirical particulars (see Figure 1). In other words, axiomatic categorisation (AC) maintains omnipresent presuppositions void of dynamic proposition: in this domain and mode of discourse, sets of vectors simulate the static state physical and engineered composition and direction of flow illustrated with the aftertreatment (A/T) case study.

\section{AXIOMATIC CATEGORISATION}

Axiomatic categorisation (AC) (Byrne et al, 2018) is a framework to interpolate information objects with low-level logic that maintains certain original concepts for more complex logical statements in higher level applications. Original concepts are practicable by transforming application models into actual implementation, with tight integration of technical transformations attainable fusing model- 
driven engineering in the application domain, rather than writing programs at the implementation level. This sub-structure is called hereby the Analytic layer (illustrated in Figure 1), the super-structure built on top is the Synthetic layer of a proposition, having truth or falsity determinable by recourse to experience. Experience in the sense that dynamic empirical determinations (sensor data) is adjudicated upon by IVHM models based on offline resources of contextual intelligence, albeit invariable, compared to sensor data, also empirical (e.g. durability test data, material and manufacturing process data, maintenance / warranty data, dimensional metrology data - in test, manufacturing and after use).

\subsection{Order before chaos}

Prior to the complexity in the synthetic layer, what is necessary is a structured presupposition before the proposition of knowledge is represented; that is, order with explicit well-structured concepts of computational assumptions. The presupposition concentrates only on static state systematised structures for computable consumption that provide the basis on which a set of original concepts with machine-understandable descriptions can describe and organise knowledge for multiple domains.

Dynamic representation in the AC framework is excluded by concentrating only on the static state, and avoiding the confusion of language permeating to manufacturing, implementation, and operational processes. This research establishes the sub-structure set of principles (a priori set of predicate rules) that interpolate low-level logic into application models for actual implementation. Low-level logic, used for the IVHM case study, makes omnipresent the composition of interdependent A/T components, the configuration of which is simulated through sets of vectors replicating functional transformations in the static state physical and engineered system. In actual implementation, interdisciplinary artefacts form vector nodes for use in higher level applications.

\subsection{First order logic}

Our axioms are based on conceptions a priori, insofar as, remove empirical particulars from experience; what remains are certain original concepts, which have their origin entirely a priori, independent from empirical particulars. AC is a first-order logic (FOL) - a collection of formal mathematical systems consistent with the axioms. Recent examples of FOL include Breiner \& Subrahmanian (2017). Processing empirical data without the presupposition of FOL-AC would be akin to channelling them as atomistic particulars with no contextual relevance. In other words, AC formally connects artefacts to other artefacts forming a community of predisposed technical transformations, thus, a community of dynamic empirical determinations in reciprocal association, thereby inheriting auxiliary causal propositions, not determined unilaterally, rather reciprocally, as in an aggregate.

\subsection{The function of axiomatic categorisation}

The number of steps needed to execute programs at the implementation level is reduced by the tight integration of technical transformations attainable using the FOL-AC framework or Analytic substructure. The function of the AC process, illustrated in Figure 2, is to channel empirical determinations towards synthetic proposition, whose truth is dependent on the composition and direction of exacting components in the physical system.

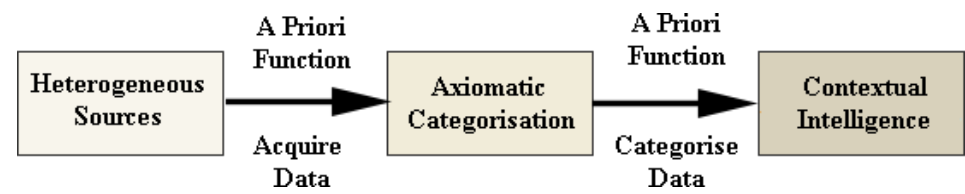

Figure 2. Analytic function model - applying static state parameters

Figure 2 illustrates the homogenisation of heterogeneous associations, to create a static state representation of functional parameters at the cyber level, consistent with the elected system of discourse. In essence, AC preserves sets of entity nodes which form vectors that represent the domain and modes of discourse, which in this case study is the A/T system.

\section{CASE STUDY: ENGINE EXHAUST AFTERTREATMENT SYSTEM}

A common engine exhaust aftertreatment (A/T) system has, in aggregate, three components - (A) the Lean NOx Trap (LNT), (B) the Diesel Particulate Filters (cDPF), and (C) the Selective Catalytic Reduction (SCR). In summary, the main system function (meet emission legislation, both in terms of 
emissions (NOx and particulates) and noise and temperature) is delivered with a number of subfunctions (written in italic in Figure 3). The LNT component is responsible for extracting HC and CO and converting NOx at low temperatures (e.g. during cold starts); the cDPF removes particulates from the flow of materials; while the SCR separates and reduces the remaining level of NOx.

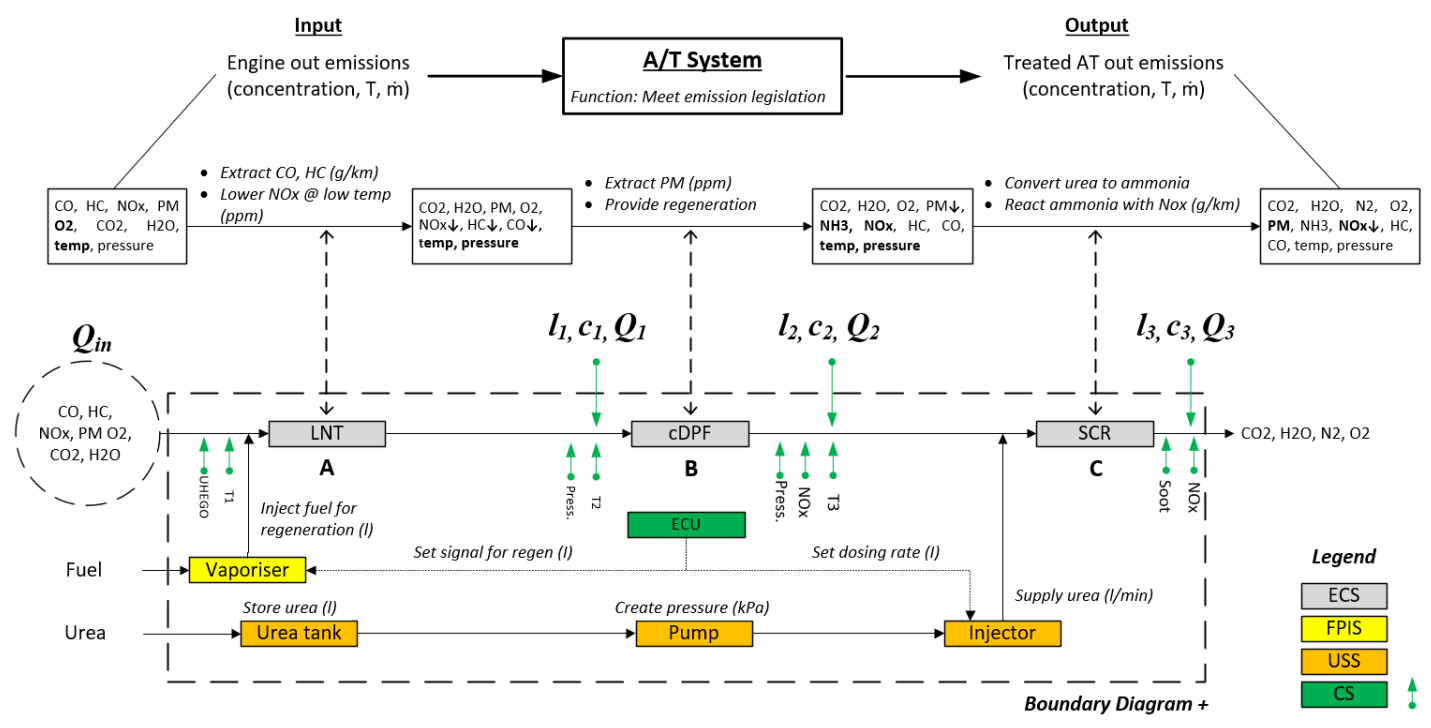

Figure 3. Aftertreament system functional representation

\subsection{Translating axiom applicablity to real world scenario}

The axioms governing this research are statements that serve as a foundation from which other, more complex statements, are logically derived. It is true that from the axioms no synthetic proposition can be made, however, to construct propositions, the axioms provide a low-level of computational logic in the form of arithmetic - the science of numbered sequences, sequentially in time. For example, under the category of relation, relations digress from subject to subject and relate to a particular discourse or mode of discourse. There is, according to Kant (1781), an a priori structure of relations that remains constant, independent from experience: so that the idea of substance is the idea that something has an enduring identity, a continuity in time; of causality, when posited, must be concurrent with or antecedent to the effect; and community is the reciprocity of action and reaction. Therefore the axiomatic sub-structure of computational arithmetic are ways of thinking about time according to rules: relate to the series in time, the content in time, the order in time, and to the complex or totality in time.

\subsubsection{The fact and the value}

Experience is not a fact, it is only a subjective value of that fact. Remove from experience empirical particulars applicable to any mode of discourse, what remains are certain original concepts (the axioms), and certain judgements derived from them which must have their origin entirely a priori, independent from experience. Product lifetime optimisation is performed by detecting, isolating and identifying faults; and then predicting the same fault parameters in simulations of replicate systems through pattern recognition, so that appropriate fault mitigation and recovery actions can be taken. Fault problems are localised into independent sub-problems, in which physically local results merge into virtually global indicators. A model of the inter-disciplinary artefact $M$ is a tuple $M=(V, F)$, where $\mathrm{V}$ is a set of variables, and $\mathrm{F}$ is a set of functional requirements - so that $\mathrm{V}$ consists of five disjoint sets, namely, the set of state variables, S; the set of parameters, $\Theta$; the set of inputs, U; the set of outputs, $\mathrm{Y}$; and the set of auxiliary variables, X. Each function $\mathrm{f}=(\varepsilon f, \mathrm{Vf}) \in \mathrm{F}$ consists of an equation $\varepsilon f$ involving variables $\mathrm{Vf} \in \mathrm{V}$. Input variables $u \in \mathrm{U}$ are known facts and correspond to (measured) sensor signals or values; and the output variables $y \in \mathrm{Y}$ correspond to (measured values of) sensor signals. Parameters $\Theta \in \Theta$ include explicit model parameters that are used in the model functions. The set $\Theta$ consists of all parameters of model functions that must be included explicitly.

The three-component A/T system model is representative of real world (the fact). Thereby, $\mathrm{f} 1$ = main system function of the $A / T$ system (i.e. meet emission legislation). For each component $i \in\{A, B, C\}, l i$ denotes the level of particulate elements of that component. $t i$ denotes the temperature levels between component, and $Q i$ denotes the volumetric flow rate out of components. For adjacent components $i$ and $j$, 
Qij denotes the flow rate in the connecting pipe, and Qin is the inflow into the LNT component from the diesel engine. The full structural decomposition is leveraged for efficient and scalable implementation of different functions of physical and engineered systems using the relation axioms.

\subsubsection{The category of relation}

The framework for the category of relation upholds original concepts that are practicable by transforming application models into actual implementation, with the tight integration of technical transformations attainable using model-driven engineering in the application domain, rather than writing programs at the implementation level. Technical transformations have the processing acumen to characterise the relations between inter-disciplinary artefacts as categorical, hypothetical, or disjunctive. Three axioms define the category of relations of artefacts to each other, conceptual or concrete.

Axiom 1: Inheritance or subsistence is the permanence of the real in time, the idea of substance is the idea that something 'is', it has an 'enduring identity', a continuity in time (Kant, 1781).

The concept of substance is a key principle of the permanence of certain constructs. The underlying representation of the physical system at the cyber level is substance. All that pertains to the objective material world can be perceived only as a determination of substance. Consequently, the permanent, in relation to which alone can all relations of time be determined, is substance, that is, that which is the underlying layer of all change, remains ever the same. For example, a posteriori propositions are determinable by the composition of physical components, which are substances, and permanent (to a degree of categorical, hypothetical, or disjunctive). Consequently time, in which all changes must be perceived, is that, in which succession and coexistence can be represented only as determinations thereof. It follows then that the arrangement of physical components is the permanent and the underlying representation of time in general, and in which all changes or coexistence can be perceived by means of empirical determination.

Axiom 2: Causality of a thing as the real which, when posited, is always followed by something else, the cause must be the concurrent with, or antecedent to the effect (Kant, 1781).

This axiom executes an explicit if/else condition. For example: (i) the causality of a thing is concurrent, when posited in component B (i.e. cDPF), only if the effect also occurred in component B; else, (ii) if the effect of a thing when posited in component $\mathrm{B}$ is antecedent, then the cause must occur outside the conceptual boundary of component B i.e., the cause occurred in either component A (i.e. LNT) or component $C$ (SCR). In other words, the provenance of the cause and the effect must be unilateral, that is, if the cause is deemed antecedent no further aggregation is possible. Therefore, on the one hand, there is the concurrent cause to the effect, which indicates that the provenance of each is the same. On the other hand, there is the antecedent cause to the effect, which indicates in this case study that the cause of the effect in component $\mathrm{B}$, occurred in component $\mathrm{A}$ or $\mathrm{C}$.

Axiom 3: Community is the reciprocal causality of substances in respect of their events, is the coexistence of the determinations of the one with those of the other - reciprocity of action and reaction (Kant, 1781).

The triad arises from the combination of the second with the first Axioms. Thus, community is the causality of a substance, reciprocally determining, and determined by other substances (substance: otherwise known as component, entity, object, artefact, etc.). However, the conjunction of the first and second, in order to produce the third, requires a particular function, which is by no means identical with those which are exercised in the first and second. Thus, if we conjoin the instance of a cause with that of a substance, it does not follow that the instance of influence, that is, how one substance can be the cause of something in another, will be understood from that. In order to assure this accordance, observe that in every disjunctive judgement, the sphere of the judgement (that is, the complex of all that is contained in it) is represented as a whole divided into parts (i.e. the A/T system has, in aggregate, three components); and, since one part cannot be contained in the other, they are processed as co-ordinated with, not subordinated to each other, so that they do not determine each other unilaterally, as in a linear series, but reciprocally, as in an aggregate.

The original configuration is the a priori constant, and the empirical particulars are the a posteriori intermittent variables in the sphere of an instantaneous proposition. In every instance of a sphere we draw a conclusion from two given propositions. First we consider relation in entities by means of the major predicates: categorical, hypothetical, or disjunctive. Then we subsume the relation under the condition of the minor predicates: inheritance or subsistence, causality, and community. Finally we determine the relational processes by means of the predicate of the rule (the conclusion), consequently, we determine it a priori by means of the original configuration. The relation, therefore, which the 
major proposition, as the rule, represents between processing empirical determinations, constitute the different kinds of instances in which a conclusion is drawn.

\subsection{Causal assigment for failure mode effect prognostics}

The initial function of the inter-disciplinary artefact model and subsequent FOL-AC presupposition is not to execute fault mitigation and recovery actions, rather it is to document fault problems that are localised into independent sub-problems, in which physically local results merge into virtually global indicators. Since the state variables $S$ are, by definition, enough to describe the future behaviour of the system, the auxiliary variables $x \in X$ are not strictly needed, however, they make the documentation of fault patterns easier to parse, develop, and improve. Product lifetime optimisation is thereby performed by detecting, isolating and identifying faults; then predicting the same fault parameters in simulations of replicate systems through pattern recognition, so that appropriate fault mitigation and recovery actions can be taken. Negating the necessity for explicitly programmed supervisory-based Life Prediction Models (LPM), Remaining Useful Life (RUL) models, Failure Mode Effect Analysis (FMEA).

Machine learning (ML) algorithms aim to produce models that can infer useful information from implicit patterns in the data, rather than explicitly coded into the program. The measured value output variables $y \in$ $Y$ that correspond to sensor signals, over a manifold of static state representations for each component i $\in$ $\{\mathrm{A}, \mathrm{B}, \mathrm{C}\}$, represent the coexistence of the determinations of the one component with those of the other. And the homogeneous manifold that chronicles the reciprocity of action and reaction is the casual model of causal assignments. Unlike existing programs which focus on the abstraction of systems built for specific proposes, depending on the context, the system dynamically chooses suitable variants to realise defined variation points, our model requires more generalisations to assign imperative knowledge. To deduce new truths from implicit patterns in the data and have the algorithm figure out what those patterns are and use them to generate a program that can infer new data about the process that generated the data, and from that, generate Failure Mode Effect Prognostic models.

\section{DISCUSSION: BROADENING THE PRODUCT LIFECYCLE LANDSCAPE}

Technology is ubiquitous in the automotive industry, opening new channels of intellectual capital, underpinned by data as the new fuel that powers decision making across the entire product lifecycle. Broadening the lifecycle landscape is a standalone contribution to the field of knowledge.

Perhaps more financially haemorrhaging to organisations than the internal disconnect is the external disconnect between suppliers, dealerships, and any third parties with bilateral vested interests. Case examples include disproportionate cost solutions for otherwise minimal repair interventions, associated with misdiagnosis of the faults. For instance, a current challenge for the automotive powertrain systems is to evolve towards meeting the emerging demands of the shift in legislation towards "real world driving emissions" - which means that legislated tailpipe emissions have to be met on actual journeys rather than standardised reference journeys tested under lab conditions. As a scenario for the (near) future, it can be envisaged that the level of allowed tailpipe emissions will increasingly depend on the state of the environmental variables. For example, in a smart mega city, the level of allowable traffic emissions could be dynamically and differentially set in relation to current location and environmental conditions. The architecture shown in Figure 4 is designed to meet these kind of emerging demands.

This paper builds on one new channel in particular, that is, online access to on-board diagnostics in the automotive vehicle, which we believe will mature into a new virtual enterprise, extending customer relations to after the release of a product to the customer, and covering the entire product lifecycle from conceptual design, to development and manufacturing, in-use, and retirement. This service-based paradigm shift is attributable to newly envisaged remote access and control capabilities to previously inaccessible powertrain components, as follows:

1. Products in the Field - Denote individual automotive vehicles or fleets of vehicles randomly dispersed within the geographical zone of a virtual enterprise. IoT technology is a radical new component of the communication core and kernel to the inter-disciplinary artefact, that is, in particular application towards open network requirements for knowledge integration into design. Using IoT, empirical determinations (i.e. data - direct from sensor; calculated from multiple sensors; calculated from sensors and offline resources: Section 3) is disseminated throughout the AAF for activities illustrated in Figure $4-2 \& 4$. 


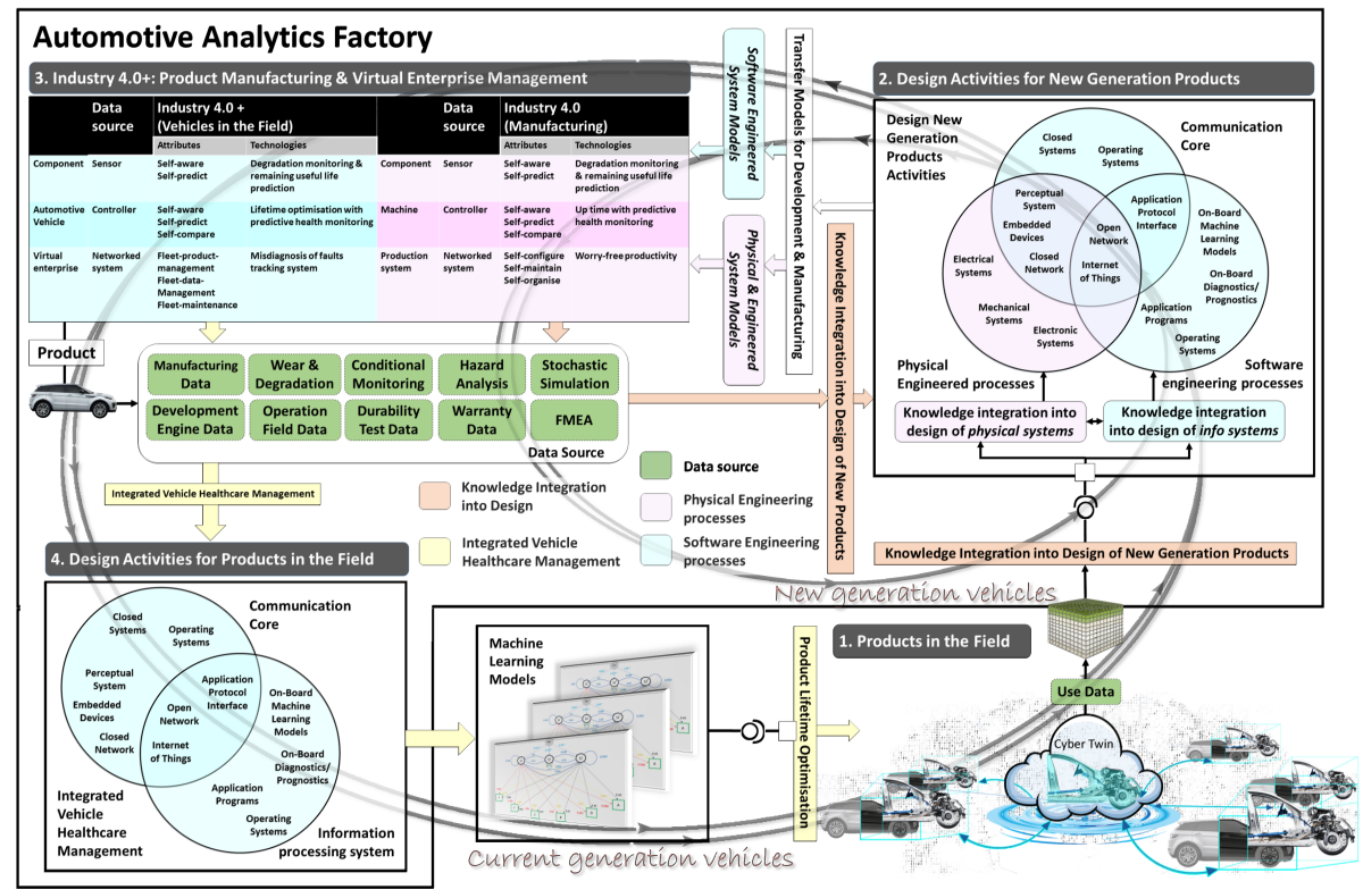

Figure 4. The Automotive Analytics Factory Architecture

2. Design Activities for New Generation Products - in the AAF, data is constantly being recycled. Multidisciplinary collaboration is needed for the continuous and iterative design and development of both new generation products (Figure 4 - 2), and front-end software solutions for current generation products (Figure 4 - 4) i.e. IVHM models. The paper addresses the multidisciplinary design problem with the conceptual inter-disciplinary artefact: a trinity of the physical and engineered component; the information processing entity; and the communication apparatus at their interface - each domain and sub-domains are represented in the Venn diagram (Figure 4 - 2).

3. Industry 4.0+: Product Manufacturing \& Virtual Enterprise Management - Industry 4.0+ extends automation and monitoring services beyond the conceptual boundary of the manufacturing facility to automotive vehicles in the field. Figure 4 proposes this novel AAF architecture, designed to:

- Enhance the operational phase of the products lifecycle using lifetime optimisation models for as close to real time IVHM of current generation automotive vehicles in the field.

- Enhance interconnect ability and intellectual capital for business, design, manufacturing, and retirement processes, that will help industry make better, faster and more informed decisions through knowledge integration into design and development of new generation products.

More traditional Industry 4.0 applications, which are mainly deployed and confined to manufacturing facilities, are not discussed in this paper (see Wollschlaeger et al, 2017; Hehenberger et. al., 2016).

4. Design Activities for Products in the Field - The main contribution of the research presented in this paper is the definition of the concept of an inter-disciplinary artefact, composed of multidisciplinary constituents. The proposed approach aims to construct first an Analytic sub-structure (AC) based on certain original concepts and certain judgements derived from them that must have their origin entirely a priori, independent from empirical particulars. The super-structure built on top we call the synthetic layer of a proposition, having truth or falsity determinable by recourse to experience. Experience in the sense that dynamic empirical determinations (sensor data) is adjudicated upon by IVHM models - developed offline in the Design phase of the AAF (Figure 4 - 4), transferred online over the air to products in the field (Figure 4 - 1), and thereafter executed offline for localised computer consumption in independent products.

In conclusion, this paper has introduced a coherent argument for a conceptual inter-disciplinary artefact to address inter-disciplinary factions, and demonstrated that the Axiomatic Categorisation provides a framework for the development of an object ontology. The impact of this concept within the automotive product lifecycle has been argued and illustrated with reasoning for the substructure architecture design for the Automotive Analytics Factory, as the meta-structure for automotive CPS lifecycle management. 


\section{REFERENCES}

Ally, A.F. and Ning, Z. (2015), "What the current system development trends tell us about systems development methodologies: toward explaining SSDAM, agile and IDER() methodologies", Journal of Applied Computer Science \& Mathematics, Vol. 19 No. 9, pp. 9-15.

Breiner, S. and Subrahmanian, E. (2017), “A Category of Design Steps”, 21st ICED 17: Design Theory and Research Methodology, Vancouver, Canada, 21.-25.08.2017.

Bylander, T. (1988), "Qualitative Representation of Behaviour in the Medical Domain”, Computers and Biomedical Research No. 21, pp. 367-380.

Byrne, T.J., Campean, F. and Neagu, D. (2018), “Towards a framework for engineering big data: An automotive systems perspective", International Design Conference - Design 2018. http://dx.doi/org/10.21278/idc.2018.0490

Dori, D. (2009), Object-Process methodology for Structure-Behaviour Co-Design.

Dori, D. and Sillitto, H. (2017), "What is a System? An Ontological Framework", Systems Eng, Vol. 20 No. 3 , pp. 207-219.

Eisenbart, B., Mandel, C., Gericke, K. and Blessing, L. (2015), "Integrated function modelling: comparing the IFM framework with SysML", Proc. 20th Int. Conf. Engineering Design (ICED15), Milan, July 27-30.

Goel, A., Gomez, A., Grue, N., Murdock, W., Recker, M. and Govindaraj, T. (1996), "Towards design learning environments- explaining how devices work", Proc. Int. Conf. Intelligent Tutoring Systems, Montreal.

Hehenberger, P., Vogel-Heuser, B., Bradley, D., Eynard, B., Tomiyama, T. and Achiche, S. (2016), "Design, modelling, simulation and integration of cyber physical systems: Methods and applications", Computers in Industry, Vol. 82, pp. 273-289.

Hirtz, J., Stone, R.B., McAdams, D.A., Szykman, S. and Wood, K.L. (2002), “A functional basis for engineering design", Research in Engineering Design, Vol. 13 No. 2, pp. 65-82.

Kant, I. (1781), "Critique of Pure Reason". Cosimo Classics, London”, England.

Rajkumar, R.R., Lee, I., Sha, L. and Stankovic, J. (2010), “Cyber-Physical Systems-The Next Computing Revolution", 47th Design Automation Conference, pp. 731-736.

Recker, J. and Niehaves, B. (2008), "Epistemological Perspectives on Ontology-based Theries for Conceptual Modelling", Applied Ontology, Vol. 3 No. 1-2, pp. 111-130.

SAE International. Integrated Vehicle Healith Management, Online. Available at: (https://www.sae.org/publications/collections/content/sub-std-00017/. Assessed: 16/12/2016.

Stankovic, J.A. (2014), "Research Directions for the Internet of Things", IEEE IoT Journal, Vol. 1 No. 1, pp. 3-9.

Umeda, Y., Takata, S., Kimura, F., Tomiyama, T., Sutherland, J.W., Kara, S., Herrmann, C. and Duflou, J.R. (2012), "Toward integrated product and process life cycle planning-An environmental perspective", CIRP Annals, Vol. 61 No. 2, pp. 681-702.

Umeda, Y. and Tomiyama, T. (2015), "Development of function modelling and its application to selfmaintenance machine", In: A. Chkrabarti and U. Lindemann, (Ed.), Impact of Design Research on Industrial Practice, Springer-Verlag, Berlin, pp. 311-319.

Wand, Y. and Weber, R. (1990), “An ontological model of an information system”, IEEE Transactions on Software Engineering, Vol. 16 No. 11, pp. 1282-1292.

Wand, Y., Storey, V.C. and Weber, R. (2000), "An Ontological Analysis of the Relationship Construct in Conceptual Modelling”, CAN Transactions on Database Systems, Vol. 24 No. 4, pp. 494-528.

Wand, Y. and Weber, R. (2004), "Reflection: Ontology in Information Systems", Journal of Database Management, Vol. 15 No. 2, pp. 3-6.

Wollschlaeger, M., Sauter, T. and Jasperneite, J. (2017), “ The future of industry communication: automation networks in the era of the internet of things and industry 4.0", Industrial Electronics Magazine, pp. 17-27.

Wynn, D.C., Eckert, C.M. and John, C.P. (2007), "Modelling iteration in engineering design", International Conference on Engineering Design.

Yan, X.Q., Li, Y., Chen, J., Li, W.Q. and Xiong, Y. (2017), “A method of implementing formalised multidisciplinary collaboration in product conceptual design process", Journal of Mechanical Engineering Science, Vol. 23 No. 18, pp. 3342-3357.

Yildirim, U., Campean, F. and Williams, H. (2017), "Function modelling using the system state flow diagram", Artifial Intelligence for Engineering Design, Analysis and Manufacturing, Vol. 31, pp. 413-435.

\section{ACKNOWLEDGMENTS}

The work presented in this paper is part of a collaborative research project on "Intelligent Personalised Powertrain Health Care", funded by Jaguar Land Rover. 